\title{
Learning in a Multi-agent System as a Mean for Effective Resource Management
}

\author{
Bartłomiej Śnieżyński and Jarosław Koźlak \\ AGH University of Science and Technology, Institute of Computer Science \\ Kraków, Poland \\ $\{$ sniezyn, kozlak\}@agh.edu.pl
}

\begin{abstract}
In this paper symbolic, supervised learning is used in a multiagent system for resource management. Environment is a Fish Bank game, where agents manage fishing companies. Rule induction is applied to generate ship allocation and cooperation rules. In this article system architecture and learning process are described and experimental results comparing performance of several types of agents are presented. The results obtained confirm that applying a supervised learning algorithm in a multi-agent system may improve resource management.
\end{abstract}

\section{Introduction}

One of the main problems in a modern society is a problem of efficient resource management. It may concern different domains like logistics, ecology, production planning or computer network designing. The management is especially difficult for common, renewable resources, i.e. the ones whose quantity in the environment increases until reaching a given maximum value, and the speed of this increase depends on the quantity of resources.

An exhaustion of common resources can be considered as an example of a crisis situation, which makes it impossible to regenerate them in the future and, as a consequence, makes the functioning of entities, which need these resources, impossible. Hence, it is important to guarantee a resource consumption on the appropriate level, and to minimize the probability of crisis situations arising as well as their scale.

The goal of our work is to provide a strategy of resource management and to guarantee that a system will stay in a safe state, i.e. the one, which does not lead to resources exhaustion.

To analyze such situations it is useful to apply multi-agent approach, which allows for modelling of activities of autonomous, rational entities - agents, which cooperate or compete one with another. Agents try to realize theirs goals in a way that is as good as possible, whereas a cooperation may be necessary to avoid an exhaustion of resources and appearing a crisis situation. The cooperation may occur as an auto-modification of actions concerning resources consumption performed by an agent or by negotiations and contracts. 
One of the main problems in the development of such systems is designing an appropriate strategy for resource allocation and determination, when negotiations and contracts should be introduced and which character they should have. Applying learning algorithms allows to overcome such problems. One can implement an agent that is not perfect, but it improves its performance.

In this paper results of application of symbolic, supervised learning in a multiagent system are presented. As an environment Fish Bank game is used 1]. It is a simulation where agents run fishing companies that must decide how much, and where to fish.

In the following sections learning in the multi-agent systems is described, developed system and a learning process used is presented, and experimental results are analyzed.

\section{Learning in Multi-agent Systems}

The problem of learning in multi-agent systems may be considered as a union of research on multi-agent systems and on machine learning. Machine learning focuses mostly on research on isolated process performed by one intelligent module. The multi-agent approach concerns the systems composed of autonomous elements, called agents, whose actions lead to the realization of given goals. In this context, learning is based on the observation of the influences of activities, performed to achieve the goal by an agent itself or by other agents. Learning may proceed in a traditional - centralized (one learning agent) or decentralized manner. In the second case more than one agent is engaged in the learning process [2].

The learning process is strictly associated with such aspects of agents as reasoning and decision making. The most popular learning technique in multiagent systems is reinforcement learning that allows to learn what action should be executed in a current situation. Other techniques can be also applied. Learning process can be based on the symbolic knowledge representation (rules, decision trees), neural networks, models coming from game theory as well as optimization techniques (like the evolutionary approach, tabu search etc.).

An important feature of cooperative learning is the necessity of the interactions between agents using a suitable protocol. Thanks to these interactions, an agent may acquire additional knowledge useful to its decision making and allowing it to make a common decision in accordance with the preferences of the agents. An example may be a solution presented in [3]. Here, agents may come forward with proposals, which are expressed by an additional parameter which describes the confidence of the agent about them. In their response, the other agents participating in the negotiation process may accept the proposal (confirm), reject it (disagree), propose modifications (modify) or do not have an opinion on the subject (no opinion). In each case, except the last one, agents also declare their confidence concerning their evaluation. 


\section{System Description}

Although Fish Banks game is designed for teaching people effective cooperation in using natural resources [4] it suits to using in multi-agent systems very well [1,5. In this research the game is a dynamic environment providing all necessary resources, action execution procedures, and time flow (game rounds). Each round consists of the following steps: ships and money update, ship auctions, trading session, ship orders, ship allocation, fishing, and fish number update.

Agents represent players that manage fishing companies. Each company aims at collecting maximum assets expressed by the amount of money deposited at a bank account and the number of ships. The company earn money by fishing at fish banks.

Environment provides two fishing areas: coastal and a deep-sea. Agents can also keep their ships at the port. Cost of fishing at the deep-sea is the highest. Cost of staying at port is the lowest but such ship does not catch fish.

Initially, it is assumed that the number of fish in both banks is close to the bank's maximal capacity. During the game the number of fish in every bank changes according to the following equation:

$$
f_{t+1}=f_{t}+b f_{t}\left(1-\frac{f_{t}}{f_{\max }}\right)-C_{t},
$$

where $f_{t}$ is a fish number at a time $t, b$ is a birth rate (0.05 value was used in experiments), $f_{\max }$ is a maximum number of fish (equal 4000 for a deep sea, and 2000 for a coastal area), $C_{t}=n c_{t}$ is a total fish catch: $n$ is a number of ships of all players sent to the bank, and $c_{t}$ is a fish catch for one ship at the time $t$ :

$$
c_{t}=c_{\max } w_{t} \sqrt{\frac{f_{t}}{f_{\max }}},
$$

where $c_{\max }$ is a maximal catch (equal 25 for a deep sea, and 15 for a coastal area), and $w_{t}$ is a weather factor at a time $t$, which is a random number between 0.8 and 1.0. As we can see, at the beginning of game, when $f_{t}$ is close to $f_{\max }$, fishing at the deep sea is more profitable.

Usually exploration overcomes birth and after several rounds the fish number can decrease to zero. It is a standard case of "the tragedy of commons" 6]. It is more reasonable to keep ships at the harbor then, therefore companies should change theirs strategies.

Three types of agents are implemented: learning agent, predicting agent, and random agent. The first one uses experience to allocate ships, the second one uses previous fishing results to estimate values of different allocation strategies, third one allocates ships randomly. All types of agents may observe the following aspects of the environment: new ships that they receive from a shipyard, money earned in the last round, ships allocations of all agents, and fishing results $\left(c_{t}\right)$ for a deep sea and an inshore area. All types of agents can execute the following two types of actions: order ships, allocate ships. 
Order ships action is currently very simple. It is implemented in all types of agents in the same way. At the beginning of the game every agent has 10 ships. Every round, if it has less then 15 ships, there is $50 \%$ chance that it orders two new ships.

Ships allocation is based on the method used in [1. Allocation strategy is represented by a triple $(h, d, c)$, where $h$ is the number of ships left in a harbor, $d$ and $c$ are numbers of ships sent to a deep sea, and a coastal area respectively. Both types of agents generate a list of allocation strategies for $h=0 \%, 25 \%, 50 \%, 75 \%$, and $100 \%$ of ships that belong to the agent. The rest of ships $(r)$ is partitioned; for every $h$ the following strategies are generated:

1. All: $(h, 0, r),(h, r, 0)$ - send all remaining ships to a deep sea or coastal area,

2. Check: $(h, 1, r-1),(h, r-1,1)$ - send one ship to a deep sea or coastal area and the rest to the other,

3. Three random strategies: $(h, x, r-x)$, where $1 \leq x<r$ is a random number - allocate remaining ships in a random way,

4. Equal: $(h, r / 2, r / 2)$ - send equal number of ships to both areas.

The random agent allocates ships using one of strategies chosen by random. Learning agent does the same in the first game, but in the following games it chooses strategy with the highest rating. Strategy rating is generated using rules that allow to classify allocation as good or bad taking into account allocation parameters and environment parameters (fish catch at the deep sea and at the coastal area in the previous round).

Every strategy $s$ gets a value $v$ according to the formula:

$$
v(s)=\alpha \operatorname{good}(s)+\operatorname{bad}(s),
$$

where $\operatorname{good}(s)$ and $\operatorname{bad}(s)$ are numbers of rules, which match the strategy and current environment parameters, with consequence good and bad, respectively, and $\alpha$ is a weight representing importance of rules with consequence good. The rules are learned using agent experience (see section 4).

If there is more then one strategy with the same value, one occurring earlier in the list is chosen.

Predicting agent uses the following formula to estimate value of the strategy:

$$
v(s)=\operatorname{income}(s)+\eta \operatorname{ecology}(s),
$$

where income $(s)$ represents prediction of the income based on the previous fishing results, ecology $(s)$ represents ecological effects of the action $s$ (the value is low if fishing is performed in the area with low fish population), and $\eta$ represents importance of the ecology factor.

Software used in experiments is written in Prolog, using Prologix compiler [7. Every agent is a separate process. It can be executed on a separate machine. Agents communicate with the environment using Linda blackboard. 
Table 1. Predicates used to describe the event

\begin{tabular}{lll}
\hline Predicate & Argument values & Description \\
\hline rate $(\mathrm{R})$ & good, bad & Rating of the allocation strategy \\
harbor (N) & $100 \%, 75 \%, 50 \%, 25 \%, 0 \%$ & Fraction of ships left in a harbor \\
alloc (A) & $100 \%-0 \%, 75 \%-25 \%, 50 \%$ - Allocation: ship fraction sent to \\
& $50 \%, 25 \%-75 \%, 0 \%-100 \%$ & deep-ship fraction sent to coastal \\
prevCatchDeep (D) & integer numbers & Number of fish cought by every ship \\
& & on a deep sea \\
prevCatchCoastal (C) & integer numbers & Number of fish cought by every ship \\
& & on a coastal area \\
\hline
\end{tabular}

\section{Learning}

To support learning AQ21 program is used 8. It is a machine learning software that allows to generate attributional calculus rules for given examples. In a standard mode it produces a complete and consistent description of the data, but it can also provide rules that are not complete and/or consistent. The main advantage of this program is that generated rules are not ordered and the knowledge is easy to interpret for human what makes experimental results easier to check and can be useful in Fish Bank application to teach people. Of course, other methods of classifier learning can be used.

\subsection{Ship Allocation Learning}

The AQ21 program generates a classifier that is used to rate ship allocation strategies. Input attributes for the classifier are allocation parameters (ratios of ships that are kept in a harbor, sent to the deep sea, and sent to a coastal area) and environment parameters. Target attribute is a rating of the allocation in a given environment state. It has two values: good and bad. Predicates used to describe the event are presented in Tab. 1.

Training events are generated from agents observations. Every round the learning agent stores ship allocations of all agents, and fish catch in the previous round. The strategy of an agent with the highest income is classified as good, and the strategy of an agent with the lowest income is classified as bad. If in some round all agents get the same income, none strategy is classified, and as a consequence, none of them is used in learning.

At the end of each game the learning agent uses events, which were generated during all games played so far, to learn a new classifier that is used in the next game.

\subsection{Cooperative Learning}

The next step in model development is to introduce cooperative learning of agents to avoid depleting of fish. There are three main aspects of negotiations that are considered: 
- selection of the time point when negotiation is performed. If an agent discovers that its situation is worse than in a previous tour, it starts a negotiation;

- selection of a proposal to be negotiated. The selection may be performed by random with preference to decision which proved to be the most advantageous in the most of situations or for the most situations in similar cases;

- decision on whether the negotiated proposition should be accepted or rejected. Agents evaluate a list of proposals (each agent accepts or rejects them) and then select the one with the highest degree of acceptance. If an agent does not have any reason to accept or reject negotiation results (i.e. does not know if the consequences of a particular condition is good or bad for it) then it makes a decision by lot. Proposal has to get a majority of the agents to be valid. At the end of game an agent assesses whether the accepted proposition improved or worsened its situation and classifies the proposal as good or bad for the given system state.

To represent the agent situation, the wealth of the agent, numbers of fish on the banks, and/or trends concerning the change of wealth or the change of the number of fish caught should be taken into account.

These attributes are used to generate rules that are used to rate proposals. Leaning rules after a game can improve negotiation strategies. As for ship allocation learning, AQ21 learning program can be applied.

\section{$5 \quad$ Experimental Results}

To test how learning changes an agent performance four series of experiments were performed. Four agents took part in every series. Every series consisted of ten simulations, and each simulation consisted of the sequence of 15 games.

In the first series there were three random agents and one learning agent (with weight $\alpha=1$ ). Performance of the agents measured as a balance at the end of every game is presented in Fig. 1 1 (a).

In the second series one learning $(\alpha=1)$, one predicting and two random agents were used. Performance of agents is presented in Fig. 1. (b).

In the third and fourth series there were two learning and two random agents. In both series, the first learning agent (LA1) has $\alpha=1$. In the former series, presented in Fig. 1 1 (c), second learning agent (LA2) has $\alpha=2$, in the latter, presented in Fig. 1 1 (d), LA2 has $\alpha=3$.

In all experiments average balance of the learning agents grows with the agent's experience, while performance of the predicting agent decreases slightly (because of the learning agent competition). Performance of random agents generally doesn't change.

Examples of rules learned are presented in Fig. 2, Capital letters represent variables that can be unified with any value. Predicate member checks if its first argument belongs to the list that is a second argument. It is used to represent an internal disjunction (expression of the form $x=v_{1} \vee v_{2} \vee \ldots \vee v_{n}$ ). Remaining predicates are explained in the previous section. These rules (in the form of clauses) can be interpreted in the following way. 
(a)

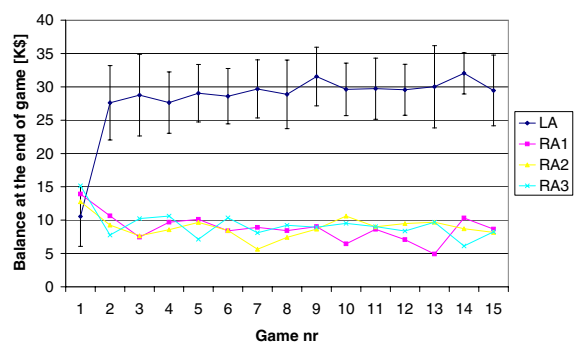

(c)

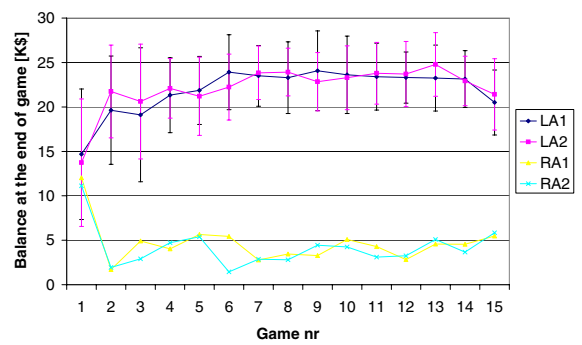

(b)

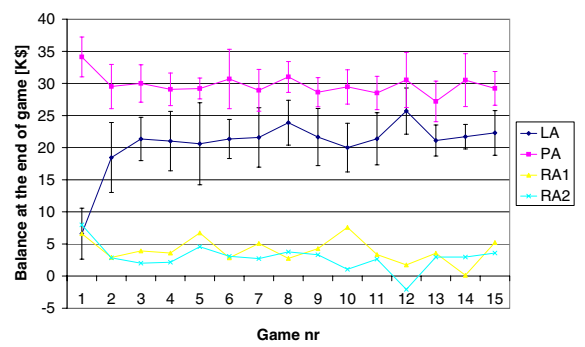

(d)

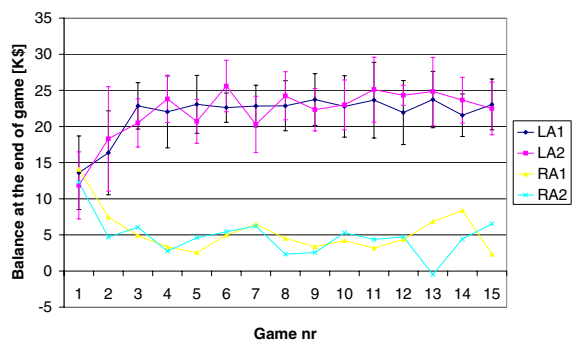

Fig. 1. Comparison of performance of learning agents (LA, LA1, LA2) and other agents using random strategy of ship allocation (RA1, RA2, RA3) or prediction (PA); values for learning and predicting agents are presented with the standard deviation

(a)

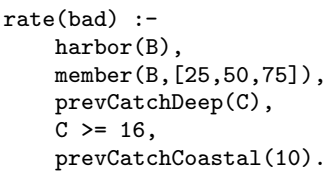

(b)

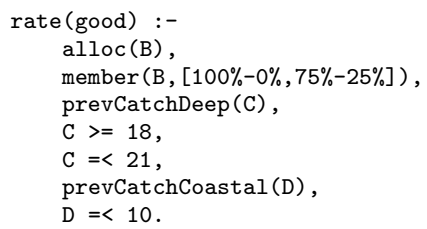

Fig. 2. Examples of rules (in the form of Prolog clauses) learned by the agent

Clause (a): it is a bad decision to keep at a harbor 25, 50, or 75 percent of ships if previous catch at a deep sea is greater or equal to 16 , and previous catch at a coastal area is 10 .

Clause (b): it is a good decision to send $100 \%$ ships to a deep sea or $75 \%$ to a deep sea and $25 \%$ to a coastal area if previous catch at a deep sea is greater or equal to 18 , and smaller or equal to 21 , and previous catch at a coastal area is smaller or equal to 10 .

Experimental results show that the learning agent performance increases rapidly at the beginning of the learning process, when generated rules are used instead of a random choice. Next it increases slowly, because new examples do not contain any significant knowledge. The performance stabilizes at the end of the process. 
Weight $\alpha$ has no significant influence on the learning agent performance. However values greater then 1 seem to be a little bit better then $\alpha=1$.

As we can see in Fig. 1.(b), the predicting agent performs better then the learning agent. It suggests, that there is a space for improvement of the learning method. Further research is necessary to check if it is possible to learn such a good strategy.

\section{Conclusion and Further Research}

The results obtained confirm that applying a symbolic machine learning algorithm in a multi-agent system may improve resource management.

Fish Banks environment is complex enough to apply supervised learning, when direct performance feedback is available (e.g. income at the end of the round), and, if there is no such information, and feedback is available at the end of game (reinforcement learning can be used in such situation). Testing the latter case is a subject of further research.

Acknowledgments. The authors thank prof. Ryszard S. Michalski, George Mason University for providing AQ21 program, and Arun Majumdar, Vivomind Intelligence Inc. for providing Prologix system, which was used in the implementation.

\section{References}

1. Kozlak, J., Demazeau, Y., Bousquet, F.: Multi-agent system to model the fishbanks game process. In: The First International Workshop of Central and Eastern Europe on Multi-agent Systems (CEEMAS'99), St. Petersburg (1999)

2. Sen, S., Weiss, G.: Learning in multiagent systems. In Weiss, G., ed.: A Modern Approach to Distributed Artificial Intelligence. The MIT Press (1999)

3. Sian, S.: Adaptation based on cooperative learning in multi-agent systems. In Demazeau, Y., Muller, J.P., eds.: Decentralised AI (Vol. 2). (1991) 257-272

4. Meadows, D., Iddman, T., Shannon, D.: Fish Banks, LTD: Game Administrator's Manual. Laboratory of Interactive Learning, University of New Hampshire, Durham, USA (1993)

5. Sniezynski, B., Kozlak, J.: Learning in a multi-agent approach to a fish bank game. In: Multi-Agent Systems and Applications IV: Proc. of CEEMAS 2005. Volume 3690 of Lecture Notes in Computer Science. (2005) 568-571

6. Hardin, G.: The tragedy of commons. Science 162 (1968) 1243-1248

7. A. Majumdar, P. Tarau, J.S.: Prologix: Users guide. Technical report, VivoMind LLC (2004)

8. Wojtusiak, J.: AQ21 User's Guide. Reports of the Machine Learning and Inference Laboratory, MLI 04-3. George Mason University, Fairfax, VA (2004) 